\title{
The Changes of Nursing Students' Assessment Skills at a Simulated Setting: A Quasi Experimental Study
}

\author{
Yok Man Cymon Chan, Haobin Yuan \\ School of Health Sciences, Macao Polytechnic Institute, Macao, China \\ Email:ymchan@ipm.edu.mo
}

Received December $11^{\text {th }}$, 2013; revised January $11^{\text {th }}, 2014$; accepted January $18^{\text {th }}, 2014$

\begin{abstract}
Copyright @ 2014 Yok Man Cymon Chan, Haobin Yuan. This is an open access article distributed under the Creative Commons Attribution License, which permits unrestricted use, distribution, and reproduction in any medium, provided the original work is properly cited. In accordance of the Creative Commons Attribution License all Copyrights (C) 2014 are reserved for SCIRP and the owner of the intellectual property Yok Man Cymon Chan, Haobin Yuan. All Copyright (C) 2014 are guarded by law and by SCIRP as a guardian.
\end{abstract}

\begin{abstract}
Background: Structured and comprehensive assessment is critical to identify physical and psychological problems and concerns experienced by patients. Simulation can be used for training students' health assessment skills as well as communication skills. Objectives: The purposes of this study were to determine how students' health assessment skills changed in a simulated setting. Method: A quasi experimental study with one group repeated-measures design was conducted with a purposive sampling of 85 baccalaureate nursing students at one nursing school in Macao. Two qualified tutors evaluated students' health assessment skills in terms of introduction and patient identification, symptom assessment, physical examination, patient education, history inquire and communication using the same criteria in each simulation session. Results: The overall score of students' health assessment skills increased from the first session to the last session. Second-year students achieved higher overall scores of assessment skills than third-year students in some simulated scenarios significantly even though they had less clinical practice experience. Conclusions: Simulation using a human patient simulator helped students to transform knowledge and skills to assess patient condition. Tutors should promote students' intrinsic motivation for learning, develop their potential and encourage them to keep their efforts in learning.
\end{abstract}

Keywords: Assessment; Baccalaureate; Nursing Student; Simulation

\section{Introduction}

Health assessment is defined as an evaluation of the health status of an individual by performing symptom assessment, physical examination and history inquiry (Day, 2010). When nurses interact with patients and provide care, the appropriate assessment skills with obtaining a complete health history are critical to identify physical and psychological problems and concerns experienced by patients. Nurses should consider patients' educational and cultural background and their language proficiency, and pay close attention to the patient's disabilities or impairments in hearing, vision, cognitive and physical limitations during health assessment (Pan, 2012). Although assessment is an essential competency of nurses, little has been actually done by nurses in clinical settings. Secrest, Norwood, \& duMont's (2005) survey showed that although 92.5\% of physical assessment skills were taught in baccalaureate nursing program, only $29 \%$ of nurses in clinical practice actually performed those skills. Liu, Chen and Yang (2008) indicated that the assessment skill deficiencies of new nurses included lack of communication skills, incorrect physical examination technique, and lack of comprehensive assessment and analysis of patient condition. A gap may exist between what is taught in classroom and what is actually performed in nursing practice. In Macao, students usually prefer to learn practical knowledge in an organized environment. The static manikins commonly have been delivered under the direction or demonstration of teachers. Students had less opportunity to learn or practice the skills in an interactive environment. Preparation of students for a complex health care environment requires that students are educated not only in skills but also in communication and collaborative care. An ongoing concern with nursing education is how to improve students' assessment skills using appropriate strategies as well as knowledge application (Mei, He, Xie, Yang, \& Duan, 2012). Students should learn how to solve problems and practice over and over in each trial while accepting and learning from their mistakes until they become skilled. In this case, more active teaching strategies, such as role play, case study, or standardized patient teaching method, were used in course learning of health assessment and showed that the experimental group got the higher scores in both theory and skill exams than the control group who served as a traditional method (Chen, Cheng, Zhou, Feng, \& Dong, 2012; Pan, 2012; Yin et al., 2012).

Although learning by doing is a long established means for facilitating knowledge acquisition, it isn't practical to engage in skill training with real patients due to the increased protection of patient rights and safety. The complexity of the current health care systems makes it difficult to provide nursing students with sufficient clinical experiences to ensure their competency. Educators are challenged to find adequate clinical experiences for their students. Simulation provides the opportunity for the learner to practice and learn in an environment as 
close to reality as possible and allows students to construct knowledge and develop psychomotor skills in a safe environment (Sinclaire \& Fergusion, 2009). Students interact with a human patient simulator and discover critical assessment information in the same manner they would with real patients. They had an opportunity to rehearse skills in a simulated environment without fear of failure or compromising patient safety (Bambini, Washburn, \& Perkins, 2009).

As an experience learning, simulation using a human patient simulator (the SimMan) which imitates patient condition with physiological functions can be used for training health assessment skills as well as communication skills (Haidar, 2009). However, little current study discussed how to improve assessment skills and knowledge application of medical-surgical nursing using simulation in nursing students. The observational measure was used in this study to discuss about the research question as a difference in health assessment scores followed by each simulation session.

\section{Research Objective}

The objective of this study was to determine how students' assessment scores changed at a simulated setting followed by each simulation session.

\section{Method}

A quasi experimental study with one group repeated-measures (from Session 1 to Session 5) design was conducted at one nursing school in Macao. The scores measured in each simulation session were compared within each tutorial group.

\section{Samples}

Purposive sampling was used to recruit Year 2 and Year 3 baccalaureate nursing students who passed course learning in health assessment and medical-surgical nursing. Totally there were 115 students (54 in Year 2, 61 in Year 3). 90 of them (52 in Year 2, 38 in Year 3) voluntarily participated in this study. Finally 85 students (49 in Year 2, 36 in Year 3) completed all simulation sessions. Their average age was 20.24 (S.D. 1.46) years old. Second-year students had 20 weeks of clinical experience while third-year students had 30 weeks of experience.

\section{Teaching Protocol}

The 36-hour simulation training was conducted as extracurricular activities for promoting students' health assessment skills. The scenarios were designed by two qualified tutors using a human patient simulator which is a computer-controlled full-body manikin with a realistic upper airway, chest movement, variable cardiac and breath sounds and a palpable pulse. In each grade, 5 or 6 students worked in a group. Students directly interacted with a contextual patient scenario and performed health assessment adhering to principles of safety. Role play was used in "nurse-patient" communication. One student served as a patient or a family member or a friend who provided personal information while the other students would be the nurses who provided patient care. The tutors acted as facilitators to promote students' learning. Each group simulation was video-recorded for evaluating students' performance in health assessment. Table 1 showed the teaching protocol in each session.

\section{Instrument}

Based on the Day's (2010) health assessment guidelines, the health assessment evaluation rubric (NAER) (an observational measurement) was developed by two qualified tutors (see Table 2). The face validity was approved by three experts in health assessment and medical-surgical nursing. It was used to evaluate students' performance in health assessment in terms of introduction and patient identification (2 items), symptom assessment (2 items), physical examination (6 items), patient education (1 item), history inquire (1 item) and communication (2 items). It is ranked as three levels (2 excellent, 1 satisfactory, 0 needs practices). The possible score ranges from 0 to 28 . A higher score indicates better performance in health assessment (see Table 2).

In this study, the NAER was completed by two qualified tutors for assessing the performance of each group. The interrater reliabilities of the NAER were 0.818 (Session $1, P<0.01$ ), 0.814 (Session 2, $P<0.01$ ), 0.812 (Session 3, $P<0.01$ ), 0.801 (Session $4, P<0.01$ ) and 0.928 (Session 5, $P<0.01$ ).

In addition, one open-end question (what do you think of simulation in promoting your assessment skills?) was used to describe students' perceptions about the impact of simulation experience on the development of health assessment.

\section{Date Collection and Analysis}

Health assessment skills were evaluated at the end of each simulation session prior to debriefing. Two tutors completed the scoring of the NAER for each group and provided the rationale for each score assigned. Differences in interpretation were discussed and negotiated until similar rational for scores given could be verbalized. The average scores of two tutors were calculated. The repeated measures analysis of variance (RM-ANOVA) was used to assess differences of health assessment within groups as well as across time for simulation. Independent samples t-test was carried out to compare the mean scores of the NAER between Year 2 and Year 3 students. Additionally, the open-ended question was completed at the end of the last simulation session. All responses were summarized by their meanings with analysis of frequency.

\section{Ethical Considerations}

The research process was discussed by the workgroup for academic affaires and approved by the board of management of the institute. Participants were provided with a complete explanation about the objective and process of the study. The written consent from each participant was obtained. All participants were entirely voluntary and had the freedom to withdraw from the study at any time. Confidentiality and autonomy were assured. Only aggregate data would be reported.

\section{Results}

The overall score of assessment skills increased from the first session to the last session, especially in communication and symptom assessment (see Table 3). Second-year students achieved higher overall scores of assessment skills in Session 2, 4 and 5 than third-year students significantly. They presented better physical examination in each session, and better communication and patient education in Sessions 3, 4 and 5 (see Table 4). 
Table 1.

The teaching protocol in each simulation session.

Content ( Learning hours)
Session 1 Surgery care (7 hours)
A 42 -year-old woman has complained the severe pain in the lower
quadrant of the abdomen for 6 hours. She has had nausea and vomiting for
two days. She was pale and painful. She was admitted to surgery department.
The doctor ordered a complete blood picture, and renal and liver function
test, and amylase level and other blood tests. They are all in the normal range
except for his white blood count. The white blood count is $12,700 / \mathrm{mm}^{3}$.
The client was diagnosed with appendicitis and needs an emergency
appendectomy immediately.

Session 2 Chronic obstructive pulmonary disease (COPD) (7 hours)

A 68-year-old man, was diagnosed with COPD 10 years ago. He has a 40-year smoking history (is still smoking) and has been hospitalized twice due to chest infections during the last 12 months. He has trouble getting his breath. The physician prescribed the low-flow oxygen therapy. His FEV1 is $26 \%$ and FEV1/FVC is $38 \%$. $\mathrm{SpO}_{2}$ is $83 \%$, Two hours later, $\mathrm{SpO}_{2}$ is $80 \%$. The arterial blood gases are reported as $\mathrm{pH} 7.25$, bicarbonate ( $\mathrm{HCO}_{3}^{-}$) 23 $\mathrm{mEq} / \mathrm{L}, \mathrm{PaCO}_{2} 55$ mmHg, $\mathrm{PaO}_{2} 56$ mmHg.

Cuing questions

Learning activities

Assessment

What makes you think it was appendicitis?

What kinds of contingencies or

emergencies may happen at this In each session, the learning moment? activities are the followings:

How do you assess the patient's Preparation for learning: case condition? clarification followed by cuing questions, and What are the meanings of FEV1, information searching for FEV1/FVC, $\mathrm{SpO}_{2}$ and blood gas report?

Symptom assessment:

What kinds of contingencies or students explored the main

emergencies may happen at this complaints and the provoking moment? and relieving factors.

How do you assess the patient's (0.5 hour)

\section{Session 3 Gastrointestinal bleeding (7 hours)}

A 73-year-old man, presented to clinic with 2 hours of massive rectal bleeding. He had an abrupt onset of passing a large amount of red blood with the bleeding? dizziness and unsteady gait. Digital rectal examination revealed no mass or tenderness, but bright red blood coated the exam glove.

Laboratory studies: The hemoglobin level dropped from $10.4 \mathrm{~g} / \mathrm{dL}$ to 7.8 $\mathrm{g} / \mathrm{dL}$. Nasogastric aspirate produced bile-stained gastric contents but no blood. He had never undergone colon cancer screening. He had been diagnosed with chronic liver disease for 5 years. He has had no excessive alcohol or tobacco use. The physician ordered a proctoscopy in the emergency department.

\section{Session 4 Myocardial infarction (7 hours)}

A 72-year-old man, complained of acute chest pain. He was admitted to the emergency department. He had spent the afternoon cleaning out the rooms and has had chest pain for the last four hours. The pain is knife-like, unrelieved by rest.

The patient becomes restless, and sounds anxious. $\mathrm{SpO}_{2}$ is $93 \%$. The electrocardiogram (ECG) showed ST segment elevation, T wave inversion and Pathologic Q waves (duration $\geq 0.04$ seconds or $>25 \%$ of R-wave amplitude) on II, III, AVF leads. Blood tests showed high levels of serum creatine kinase (CK)-MB is $45 \mathrm{U} / \mathrm{L}$ (norm 0 - $23 \mathrm{U} / \mathrm{L}$ ).

\section{Session 5 Critical care: Trauma (8 hours)}

A 32-year-old man was injured while working in a high place. He fell and slid against a sharp stone on his way down, landing almost in a standing-up position and then slumping to the ground. He had multiple scrapes over his anterior torso and a large gash over his right anterior upper thigh (near the groin) which was bleeding profusely. His friends called an ambulance. The client became increasingly disoriented on the way to hospital. The client was admitted to the emergency room. $\mathrm{SpO}_{2}$ is $93 \%$. Skin was cold and clammy, and nail beds, palms, and mucous membranes were pale. He had multiple abrasions over his chin, neck, anterior thorax, and abdomen. A $15 \mathrm{~cm}$-long, $2.5 \mathrm{~cm}$-deep laceration was noted in the right inguinal region, extending into the right, upper thigh. condition?

\section{performed?} emergencies may happen at this moment?

How do you assess the patient's condition?

Why does the client have chest pain?

Is it the possibility of cardiac dysthymia? Why?

What kinds of contingencies or emergencies may happen at this moment?

How do you assess the patient's condition?
What kinds of contingencies or

Physical examination: Students conducted the physical examination meanwhile the results of laboratory were present. (0.5 hour)

Patient education: Students identified the abnormal signs and symptoms, and provided the clients. (0.5 - 1 hour)

s History inquires: Students took health history and managed any patient condition, such as pain, comments on breathless, vomiting, nausea, students' learning breathless, vomiting, nausea, students'
cough, thirst and hunger. (0.5 hour)

Nursing diagnoses: Based on the results of health

assessment and laboratory examination, students defined the problems which required nursing care and their priorities. (0.5 hour) Reflection: Students reflected on their performance and

What would happen after severe wrote a reflection paper. The bleeding? reflection questions are: (1)

What additional data would you What were the knowledge and collect? Why? skills you used in this session? (2) What needs to be

What kinds of contingencies or improved in the next session? emergencies may happen at this (2.5 - 3 hours)

moment?

How do you assess the patient's condition?
In each session, two tutors observed students performance and evaluated their using the health assessment evaluation rubric, and gave the

(1) assessment skills
Regarding students' written comments, students indicated that simulation enhanced their auscultation skills (70.6\%) and their abilities to prioritize the problems (78.8\%), to assess the client systematically $(76.5 \%)$, to manage contingencies and emergencies $(74.1 \%)$, to collaborate with others effectively (50.6\%). However, $71.8 \%$ students considered it was difficult to emulate the lived experience using the SimMan because of the slow response to inquiries and no facial expression.

\section{Discussion}

The findings indicated that simulation using a human patient simulator facilitated students' health assessment skills, especially in communication and symptom assessment. It is possibly caused by scenario design and learning activities in simulation. The scenarios used in this study were designed to reveal the ability of students to make sense of data, not only in how to 
Table 2.

Nursing assessment evaluation rubric

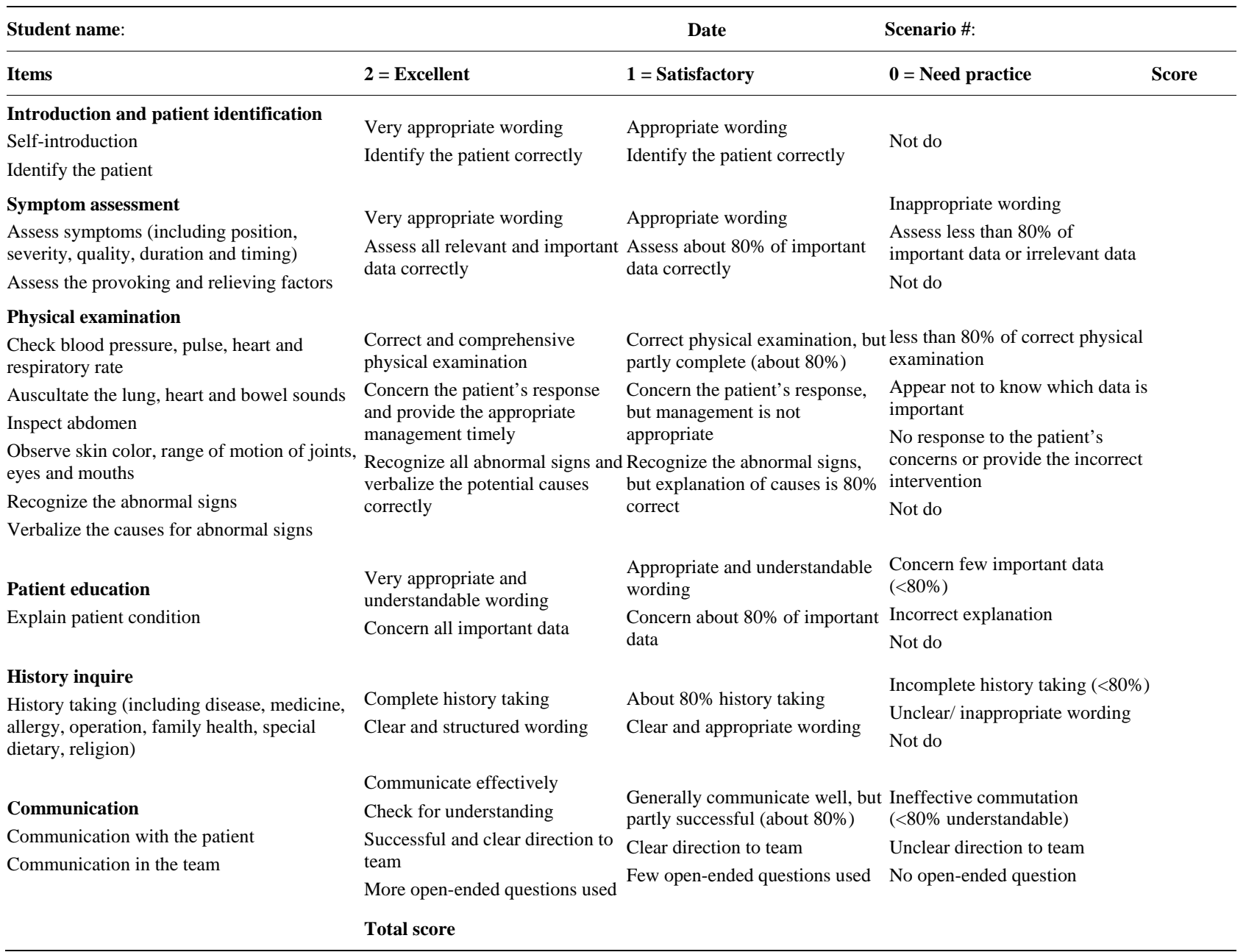

Table 3.

Changes of mean scores in nursing assessment $(\mathrm{N}=85)$.

\begin{tabular}{|c|c|c|c|c|c|c|}
\hline \multirow{2}{*}{ Nursing Assessment } & \multicolumn{5}{|c|}{ Item mean (S.D.) } & \multirow{2}{*}{$\begin{array}{c}\text { RM-ANOVA } \\
\text { Greenhouse-Geisser Value }\end{array}$} \\
\hline & Session 1 & Session 2 & Session 3 & Session 4 & Session 5 & \\
\hline Introduction & $0.77(0.55)$ & $1.46(0.39)$ & $1.60(0.36)$ & $1.62(0.40)$ & $1.49(0.59)$ & $\begin{array}{c}\mathrm{F}=44.984 \\
P=0.000\end{array}$ \\
\hline Symptom assessment & $0.91(0.37)$ & $0.67(0.37)$ & $1.01(0.34)$ & $1.30(0.42)$ & $1.50(0.38)$ & $\begin{array}{c}\mathrm{F}=73.460 \\
P=0.000\end{array}$ \\
\hline Physical examination & $0.80(0.31)$ & $1.02(0.40)$ & $1.02(0.49)$ & $1.13(0.38)$ & $1.46(0.38)$ & $\begin{array}{c}\mathrm{F}=43.231 \\
P=0.000\end{array}$ \\
\hline Patient education & $0.38(0.36)$ & $0.72(0.46)$ & $0.50(0.52)$ & $0.85(0.61)$ & $1.30(0.44)$ & $\begin{array}{c}\mathrm{F}=68.892 \\
P=0.000\end{array}$ \\
\hline History inquire & $1.26(0.63)$ & $0.95(0.48)$ & $1.00(0.63)$ & $1.01(0.47)$ & $1.12(0.45)$ & $\begin{array}{l}\mathrm{F}=5.726 \\
P=0.001\end{array}$ \\
\hline Communication & $0.65(0.28)$ & $0.72(0.25)$ & $0.79(0.27)$ & $0.89(0.41)$ & $1.09(0.38)$ & $\begin{array}{l}\mathrm{F}=4.127 \\
P=0.007\end{array}$ \\
\hline Overall score & $0.80(0.15)$ & $0.91(0.22)$ & $1.03(0.27)$ & $1.20(0.31)$ & $1.33(0.35)$ & $\begin{array}{c}\mathrm{F}=66.533 \\
P=0.000\end{array}$ \\
\hline
\end{tabular}


Table 4.

Comparison of scores of health assessment skills between Year 2 and Year 3 students in each session.

\begin{tabular}{|c|c|c|c|c|c|}
\hline \multirow{2}{*}{ Health assessment } & \multicolumn{5}{|c|}{ Mean (S.D.) } \\
\hline & Session 1 & Session 2 & Session 3 & Session 4 & Session 5 \\
\hline \multicolumn{6}{|l|}{$\begin{array}{l}\text { Introduction and patient } \\
\text { identification }\end{array}$} \\
\hline Year 2 & $0.66(0.57)$ & $1.58(0.35)$ & $1.59(0.31)$ & $1.69(0.35)$ & $1.71(0.29)$ \\
\hline Year 3 & $0.92(0.46)$ & $1.30(0.40)$ & $1.62(0.43)$ & $1.53(0.45)$ & $1.17(0.75)$ \\
\hline Independent samples t-test & $\begin{array}{l}\mathrm{t}=-2.264 \\
P=0.026\end{array}$ & $\begin{array}{l}\mathrm{t}=3.352 \\
P=0.001\end{array}$ & $\begin{array}{l}\mathrm{t}=-0.328 \\
P=0.744\end{array}$ & $\begin{array}{l}\mathrm{t}=1.921 \\
P=0.058\end{array}$ & $\begin{aligned} \mathrm{t} & =4.109 \\
P & =0.000\end{aligned}$ \\
\hline \multicolumn{6}{|l|}{ Symptom assessment } \\
\hline Year 2 & $0.74(0.32)$ & $0.71(0.59)$ & $1.00(0.36)$ & $1.49(0.28)$ & $1.62(0.22)$ \\
\hline Year 3 & $1.13(0.33)$ & $0.60(0.33)$ & $1.20(0.28)$ & $1.04(0.44)$ & $1.33(0.48)$ \\
\hline Independent samples t-test & $\begin{array}{l}\mathrm{t}=-5.497 \\
P=0.000\end{array}$ & $\begin{array}{l}\mathrm{t}=1.456 \\
P=0.149\end{array}$ & $\begin{array}{l}\mathrm{t}=-2.781 \\
P=0.149\end{array}$ & $\begin{array}{l}\mathrm{t}=5.367 \\
P=0.000\end{array}$ & $\begin{array}{l}\mathrm{t}=3.381 \\
P=0.001\end{array}$ \\
\hline \multicolumn{6}{|l|}{ Physical examination } \\
\hline Year 2 & $0.95(0.24)$ & $1.21(0.34)$ & $1.14(0.49)$ & $1.26(0.41)$ & $1.60(0.23)$ \\
\hline Year 3 & $0.60(0.26)$ & $0.76(0.33)$ & $0.86(0.46)$ & $0.96(0.26)$ & $1.27(0.46)$ \\
\hline Independent samples t-test & $\begin{array}{l}\mathrm{t}=6.437 \\
P=0.000\end{array}$ & $\begin{array}{l}\mathrm{t}=6.113 \\
P=0.000\end{array}$ & $\begin{array}{l}\mathrm{t}=2.670 \\
P=0.009\end{array}$ & $\begin{aligned} \mathrm{t} & =3.977 \\
P & =0.000\end{aligned}$ & $\begin{array}{l}\mathrm{t}=3.871 \\
P=0.000\end{array}$ \\
\hline \multicolumn{6}{|l|}{ Patient education } \\
\hline Year 2 & $0.40(0.38)$ & $0.67(0.47)$ & $0.61(0.51)$ & $1.16(0.48)$ & $1.45(0.36)$ \\
\hline Year 3 & $0.36(0.33)$ & $0.79(0.43)$ & $0.34(0.49)$ & $0.44(0.52)$ & $1.10(0.48)$ \\
\hline Independent samples t-test & $\begin{array}{l}\mathrm{t}=0.465 \\
P=0.643\end{array}$ & $\begin{array}{l}\mathrm{t}=-1.174 \\
P=0.244\end{array}$ & $\begin{array}{l}\mathrm{t}=2.398 \\
P=0.019\end{array}$ & $\begin{array}{l}\mathrm{t}=6.576 \\
P=0.000\end{array}$ & $\begin{array}{l}\mathrm{t}=3.733 \\
P=0.000\end{array}$ \\
\hline \multicolumn{6}{|l|}{ History inquire } \\
\hline Year 2 & $1.53(0.56)$ & $1.15(0.46)$ & $1.29(0.60)$ & $1.10(0.50)$ & $1.16(0.54)$ \\
\hline Year 3 & $0.90(0.54)$ & $0.68(0.36)$ & $0.63(0.45)$ & $0.91(0.39)$ & $1.06(0.29)$ \\
\hline Independent samples t-test & $\begin{array}{l}\mathrm{t}=5.149 \\
P=0.000\end{array}$ & $\begin{array}{l}\mathrm{t}=5.119 \\
P=0.000\end{array}$ & $\begin{array}{l}\mathrm{t}=5.576 \\
P=0.000\end{array}$ & $\begin{array}{c}\mathrm{t}=1.983 \\
P=0.051\end{array}$ & $\begin{array}{l}\mathrm{t}=1.180 \\
P=0.242\end{array}$ \\
\hline \multicolumn{6}{|l|}{ Communication } \\
\hline Year 2 & $0.70(0.32)$ & $0.76(0.18)$ & $0.86(0.25)$ & $1.06(0.25)$ & $1.23(0.34)$ \\
\hline Year 3 & $0.57(0.20)$ & $0.69(0.33)$ & $0.69(0.28)$ & $0.67(0.48)$ & $0.91(0.35)$ \\
\hline Independent samples t-test & $\begin{array}{l}\mathrm{t}=2.289 \\
P=0.025\end{array}$ & $\begin{array}{l}\mathrm{t}=1.326 \\
P=0.189\end{array}$ & $\begin{array}{l}\mathrm{t}=2.930 \\
P=0.004\end{array}$ & $\begin{array}{l}\mathrm{t}=4.326 \\
P=0.000\end{array}$ & $\begin{array}{l}\mathrm{t}=4.246 \\
P=0.000\end{array}$ \\
\hline \multicolumn{6}{|l|}{ Overall score } \\
\hline Year 2 & $0.80(0.12)$ & $1.00(0.13)$ & $1.08(0.25)$ & $1.36(0.25)$ & $1.47(0.20)$ \\
\hline Year 3 & $0.79(0.12)$ & $0.79(0.25)$ & $0.98(0.29)$ & $0.97(0.23$ & $1.13(0.42)$ \\
\hline Independent samples t-test & $\begin{aligned} \mathrm{t} & =0.104 \\
P & =0.918\end{aligned}$ & $\begin{array}{l}\mathrm{t}=4.743 \\
P=0.000\end{array}$ & $\begin{array}{l}\mathrm{t}=1.737 \\
P=0.086\end{array}$ & $\begin{array}{l}\mathrm{t}=7.376 \\
P=0.000\end{array}$ & $\begin{array}{l}\mathrm{t}=4.604 \\
P=0.000\end{array}$ \\
\hline
\end{tabular}

assess the patients and set priorities but also in how to provide patient education on complex topics. Students used analytic thinking and clinical reasoning processes to interpret the meanings of obtained data, and chose the appropriate response to patient condition meanwhile they also need communicate with the patient in understandable way. The interaction with the realistic scenarios enabled students to "understand" patient's feelings and realize the severity and urgency of patient condi- 
tions, and "read" the patient's responses to the intervention. This experience can help students to bridge the theory practice gap by transferring cognitive learning into practical experience. Health assessment skills were increased by assessment of the relevant data, a logical interpretation and reasoning and accurate judgments.

The reflections offered a unique way for students to critically analyze their own performance. Students engaged in introspective learning to self-correct. The reflections focused on students' primary misconceptions, anything they missed in report or other information they needed from report or the patient to act more effectively, and what they should do differently the next time while emphasizing what was correct, appropriate and safe. It allows the student to clarify their thinking and link the simulation to real situation while reinforcing specific knowledge, and to discuss how to intervene professionally in complex clinical situations (Gaberson \& Oermann, 2010). In this case, students learned from previous experience and paid close attention to patients' concerns. They assessed the relevant and important data and explained them to the patient using understandable wording as managing the contingencies and emergencies. They presented better communication skills and patient education across the time of simulation.

Previous studies reported the consistent findings. Kaddoura (2010) reported that simulation prepared new nursing graduates well to care confidently for critically ill patients, and helped them learn to make sound clinical decisions to improve patient outcomes. Zheng et al. (2010) found that students' performance was significantly improved in application of theoretical knowledge, health education and humanistic care after one-semester of simulation. More than $95 \%$ of students agreed that feedback sessions confirmed management of patients' problems, helped to develop rationale for actions (Wotton, Davis, Button, \& Kelton, 2010).

However, students indicated that the SimMan is not realistic enough. The SimMan had its own inherent limitation. It may do not duplicate the experience of working with a live patient. By responding to a situation during the scenario, the "patient" provided instant feedback; through which students saw the outcomes of their interventions. It was suggested that forthright feedback from the facilitator was needed to enhance the realism of the scenario with physical props and psychosocial interactions (Birkhoff \& Donner, 2010).

The interesting finding in this study was that second-year students achieved higher overall scores of health assessment in some sessions than third-year students. They presented better physical examination in each simulation session, and better communication and patient education in some sessions. It may be caused by the different learning effort of students. The tutors' comments showed that second-year students valued the newly learned knowledge and applied it in the simulated scenarios. They did good preparation for learning and engaged in group learning, deep discussion and reflection. They try their best to make the physical examination comprehensive while concerning the patient's response and providing the appropriate management. However, third-year students did not have a deep memory and understanding of some knowledge that they learned in their previous two years, and did not do a full knowledge review and a good skill preparation for the simulated learning. Their assessment was not comprehensive while their explanations to abnormal sign and symptoms were incorrect or ambiguous. Sometime they could not recognize some severe arrhythmias. Thereby, they got the lower scores in physical examination, patient education and communication compared with the second-year students. For ensuring the quality of learning, students should have good preparation for knowledge and skills, be self-motivated and keep responsible for their own learning. Tutors should promote students' intrinsic motivation for learning and develop their potential efforts in learning during simulation.

\section{Limitations}

The generalization of the findings was limited because a small purposive sample was recruited from one research setting. The new developed health assessment evaluation rubric was only used in medical-surgical care; the generalizability of further studies needs to be considered in other area of nursing care, such as long-term care or community care. As a confounding variable, the mixed role play of students (patients or family members and nurse) may affect the effective "nurse-patient" communication and thus influence the accuracy and scores of health assessment.

\section{Recommendations}

The performance indicators of the health assessment evaluation rubric require more research to address content and construct validity in different nursing contexts in order to more accurately reflect the current understanding of each aspect of health assessment. As transfer of skill from the simulated environment to the clinical setting is essential, follow-up studies need to be concerned with the impact of using simulation on students' performance in clinical placement.

\section{Conclusion}

Simulation using a human patient simulator offered a realistic learning environment for students to develop their health assessment skills. Most of students appreciated that simulation facilitated their knowledge application, assessment and communication skills and group collaboration, but using mannequins did not replace working with live patients. Forthright feedback from the facilitator was needed to enhance the realism of the scenario. Tutors should promote students' intrinsic motivation for learning and develop their potential and efforts in learning. The questionnaire needed to be carried out to investigate students' perceptions about the impact of simulation experience on the development of health assessment.

\section{REFERENCES}

Bambini, D., Washburn, J., \& Perkins, R. (2009). Outcomes of clinical simulation for novice nursing students: Communication, confidence, clinical judgment. Nursing Education Perspectives, 30, 79-82. http://dx.doi.org/10.3928/00220124-20100503-03

Birkhoff, S. D., \& Donner, C. (2010). Enhancing pediatric clinical competency with high-fidelity simulation. The Journal of Continuing Education in Nursing, 41, 418-423.

Chen, Y., Cheng, Y., Zhou, J. Z., Feng, M. H., \& Dong, L. F. (2012). Teaching of the course of Health assessment for vocational nursing students: Integration of theory with practice. Journal of Nursing Science, 27, 66-68.

Day, R. A. (2010). Health assessment. In R. A. Day, P. Paul, B. Williams, S. C. Smeltzer, \& B. G. Bare (Eds.), Textbook of Canadian medical-surgical nursing (2nd ed., pp. 61-89). Pennsylvania, Philly: 
Wolters Kluwer.

Gaberson, K. B., \& Oermann, M. H. (2010). Clinical teaching strategies in nursing (3rd ed.). New York, NY: Springer Publishing.

Haidar, E. (2009). Clinical simulation: A better way of learning? Nursing Management, 16, 22-23.

Kaddoura, M. A. (2010). New graduate nurses' perceptions of the effects of clinical simulation on their critical thinking, learning, and confidence. The Journal of Continuing Education in Nursing, 41, 506-516. http://dx.doi.org/10.3928/00220124-20100701-02

Liu, X. H., Chen, Y. Y., \& Yang, Y. Q. (2008). Issues and strategies in health assessment among new nurses. China Medical Herald, 5, 151152.

Mei, H., He, J., Xie, J. P., Yang, D. B., \& Duan, Y. F. (2012). Assessment simulated patients-based clinical teaching. China medical Equipment, 9, 1-3.

Pan, S. H. (2012). Teaching reform of health assessment focused on training clinical nursing ability. Journal of Higher Medical Education in Medicine (Chinese), 8, 60-61.

Secrest, J. A., Norwood, B. R., \& duMont, P. M. (2005). Physical as- sessment skills: A descriptive study of what is taught and what is practiced. Journal of Professional Nursing, 21, 114-118. http://dx.doi.org/10.3928/00220124-20100701-02

Sinclaire, B., \& Ferguson, K. (2009). Integrating simulated teaching/ learning strategies in undergraduate nursing education. International Journal of Nursing Education Scholarship, 6, 1-11.

Wotton, K., Davis, J., Button, D., \& Kelton, M. (2010). Third-year undergraduate nursing students' perceptions of high-fidelity simulation. Journal of Nursing Education, 49, 632-639. http://dx.doi.org/10.3928/01484834-20100831-01

Yin, H. Y., Xu, Z. F., Wei, X. D., Long, H., \& Nong, C. M. (2012). Application of standard patient in skill training of health assessment. Journal of Youjiang Medcial University for Nationalities, 34, 813814.

Zheng, Y. H., Jin, Y. M., Xie, X. Y., Zhong, J. Q., Su, H., \& Zhu, Q. E. (2010). Effects of school-based simulation training before pre-graduation clinical practice among nursing undergraduates. Chinese Journal of Nursing, 45, 1001-1003. 